\title{
ORAL MYOFUNCTIONAL AND ELECTROMYOGRAPHIC EVALUATION OF THE ORBICULARIS ORIS AND MENTALIS MUSCLES IN PATIENTS WITH CLASS II/1 MALOCCLUSION SUBMITTED TO FIRST PREMOLAR EXTRACTION
}

\author{
Denize Ramirez de SOUZA ${ }^{1}$, Tatiana Adamov SEMEGHINI' ${ }^{2}$, Lucio Benedito KROLL ${ }^{3}$, Fausto BERZIN ${ }^{4}$
}

1- Speech-Language Pathologist, MSc in Physiology of the Exercise, Federal University of São Paulo (UNIFESP), São Paulo, SP, Brazil. 2- DDS, PhD in Bucco-Dental Biology, Professor, Master's Degree Program in Physiological Sciences, University of Western São Paulo State, Presidente Prudente, SP, Brazil.

3- Statistician, PhD in Statistics, Professor, Master's degree Program in Physiological Sciences, University of Western São Paulo State, Presidente Prudente, SP, Brazil.

4- DDS, PhD in Anatomy, Chair Professor, State University of Campinas, Campinas, SP, Brazil.

Corresponding addres: Dra. Denize Ramirez de Souza, Avenida Washington Luis 2536, sl 401 - 19023-550 - Presidente Prudente - SP Brazil. Phone:/Fax: 1832212144 - e-mail: denize@prudenet.com.br

Received: March 28, 2007 - Modification: September 20, 2007 - Accepted: February 13, 2008

\begin{abstract}
$O$

bjective: The aim of this study was to assess the presence of oral myofunctional alterations before and after first premolar extraction in Class II/1 malocclusion patients that could endanger the long-term dental arch stability. Material and Methods: The study was performed by means of morphological, functional and electromyographic analyses in 17 Class II/1 malocclusion patients (group T) and 17 Class I malocclusion patients (group C -control), both groups with 12-30-year age range (mean age: $20.93 \pm 4.94$ years). Results: Data analyzed statistically by Student's t-test showed a significant decrease $(p<0.05)$ in the maxillary and mandibular dental arch perimeters after orthodontic treatment, but lip posture at rest did not present statistically significant differences after treatment $(\mathrm{p}>0.05)$. The Kruskal-Wallis test analyzed data from lip posture (orbicularis oris muscle) at rest and during swallowing, as well as the mentalis muscle behavior during the above-mentioned function, not showing statistically significant differences ( $>0.05$ ) after treatment (groups $\mathrm{T} 1$ and $\mathrm{T} 2$ ). However, group $\mathrm{T}$ differed significantly from group $\mathrm{C}(\mathrm{p}<0.05)$. Lip posture during swallowing showed statistically significant differences $(\mathrm{p}<0.05)$ for subjects submitted to orthodontic therapy when compared to data acquired before the treatment. The electromyographic analysis confirmed these data. Conclusions: Found myofunctional alterations observed after the orthodontic treatment in Class II/1 malocclusion seemed to jeopardize the long-term orthodontic stability, making recurrence possible.
\end{abstract}

Key words: Orbicularis oris muscles. Mentalis muscle. Electromyography. Oral myofunctional evaluation. Tooth extractions.

\section{INTRODUCTION}

Muscles carry out a modeling performance on bones and dental arches, whether at rest or during stomatognathic system function. Since our bones and teeth react to forces that work on them, consequently, strong and well-developed muscles are related to bones in good shape.

When these forces are unbalanced, they misact over the occlusion and the teeth loose their correct axial inclination while searching for other balanced position, thereby harming the dental arches and leading to dentofacial distortions. A good example of this process is Class II subdivision 1 (Class II/1) malocclusion, in which a retrognathic skeletal profile is observed $^{5}$. In these cases, the excessive overjet makes facial muscles adapt due to abnormal contraction patterns, which may cause a larger growth of the lower facial third with consequent facial elongation ${ }^{25}$. In addition, there is a combination of dental and skeletal factors that lead to an occlusal dissonance characterized by a deficiency between both bone bases, in a facial anteroposterior direction ${ }^{5,16}$.

The exaggerated lip eversion on the maxillary incisor makes lip seal difficult ${ }^{5,8,17}$. The discrepancy between bone bases leads to hypofunction and shortening of upper and lower lips, with eversion, and closure occurs on the palatal surface of the maxillary incisors, leading to mentalis muscle hyperfunction, as it contributes to the increase of the lower portion of the orbicularis oris muscle ${ }^{5}$.

In cases of orthodontically treated maxillary protrusion, there is an upper lip muscle tension increase towards the mentalis muscle when lips are closed ${ }^{3}$. People with Class II/ 
1 malocclusion usually have the effort of making lips approach during swallowing ${ }^{5}$, with the lower lip touching the palatal surface of the maxillary incisors, which causes lip hyperfunction ${ }^{5,8,23}$. Swallowing this way may maintain or increase the overjet, and even cause overcontraction of the mentalis muscle, in an attempt to close ${ }^{5}$. The subject moves the jaw downwards and forwards to make the contact between lips possible ; however, after Class II/1 orthodontic treatment, the lower lip no longer touches the palatal surface of the maxillary incisors during the above mentioned function ${ }^{23}$. Functions like swallowing and suctioning require the effective help from labial muscles in order to make closure and labial protrusion possible ${ }^{24}$. Therefore, the analysis of these functions is of great importance so that differences in muscle activities can be identified and correlated to the occlusion.

Electromyography investigates muscle function by capturing the electric signals coming from the muscles, and thus constitutes an important means to study muscle dynamics during the functions of the stomatognathic system $^{4}$.

In several Class II/1 patients, aiming to obtain a harmonic balance among tooth size, dental arch length and facial profile, the orthodontist measures the total bone length of each arch plus the width of teeth, and established the diagnosis with first molar extraction being part of the treatment plan. This procedure, however, can promote changes in patients with soft tissue profile ${ }^{3,10}$ due to dental retraction ${ }^{3,6,12,18,19}$.

Exactly because of the intimate relationship among muscles' actions, bones and dental arches, speech-language pathology meets dentistry, as both specialties deal with the functional balance of the stomatognathic system. Therefore, the aim of this study was to assess, by clinical and electromyographic examinations, the existence of myofunctional alterations in the orbicularis oris and mentalis muscles of Class II/1 malocclusion subjects, before and after orthodontic treatment, which could endanger the long-term dental arch stability. Class I malocclusion subjects served as a control group.

\section{MATERIALAND METHODS}

The research protocol was independently reviewed and approved by the Ethics in Human Research Committee of the University of Western São Paulo State and the study design was approved (protocol \#012/ 001).

Thirty-four volunteers were selected and allocated to two non-randomized groups $(\mathrm{n}=17)$ : a treatment group (group T) and a control group (group C). Group T was treated orthodontically in the Orthodontics Research Center (CEO) in Presidente Prudente, SP, Brazil. The inclusion criteria for group T were: a) to be from both genders; b) age between 13 and 30 years; c) to have Class II/1 malocclusion (as determined by CEO cephalometric analysis protocol) with need of mandibular and/or maxillary first premolar extraction. The inclusion criteria for Group $\mathrm{C}$ were: a) to be from both genders; b) age between 13 and 30 years; c) to have Class I malocclusion without tooth crowding. The exclusion criteria for both groups were to have: a) missing or lost tooth; b) high prevalence of cavitations; c) anterior or posterior crossbite; d) open bite; e) dental prosthesis; f) general health problems; g) do not agree with the regulations of the informed consent form for participation in human subject research.

\section{This study was performed in two stages}

First stage - (group C and group T, being performed after clinical examination and clinical interview and before orthodontic treatment start): a) Oral myofunctional evaluation, verified by visual inspection of lip posture at rest, which was defined as closed or opened; in the latter situation, the opening length was measured with a manual caliper (in mm); b) Water swallowing (20 mL), during which it was verified the overuse of perioral muscles (orbicularis oris and mentalis muscles) and the presence of lower lip interposition against the maxillary incisors during swallowing; c) Measurement in millimeters on plaster models using the Arch Measuring Instrument ("Amigo" - The Company) for analysis of the mandibular and maxillary dental arch perimeters. For group T, data collected from this stage was treated as group T1 data (before orthodontic treatment). Orthodontics was performed during at least a 24-month period for each patient and the first premolars were extracted during the course of the orthodontic therapy, according to the treatment plan.

Second stage - (group T only, performed 1 month before completion of the orthodontic treatment and removal of brackets): the oral myofunctional evaluation was repeated. Data collected from this stage was treated as group T2 data (after orthodontic treatment).

In addition, electromyographic evaluation of the activities of the upper (UOM) and lower (LOM) orbicularis oris muscle was performed in both groups. Data was acquired in root medium square (RMS) and expressed as $\mu \mathrm{V}^{4}$

The muscle electric potential was captured by a signalconditioning module (SCM) (1000-V2) (Lynx Electronics Technologies, São Paulo, SP, Brazil) to which passive electrodes were connected. The analogical signals were stored on the SCM and filtered with a range of cutting frequency from $20 \mathrm{~Hz}$ to $500 \mathrm{~Hz}^{1}$ through a butterworth analogical filter and 600 times final gain amplification. The system had analog-to-digital (A/D) signal conversion plate of 12-bit resolution and support DMA (direct memory access) with $2000 \mathrm{~Hz}$ sampling frequency and software for data acquisition and storage (Lynx Electronics). Signal processing was performed with MATLAB 5.0 software to obtain the RMS ${ }^{4}$.

The volunteers were oriented to comfortably keep their backs on the chair back, maintaining their feet parallel, touching the floor, and their heads positioned with the Frankfurt plan parallel to the floor. The volunteers had their skin cleaned with $70 \%$ alcoholic solution to avoid any interference that could decrease the impedance. For each studied muscle, a pair of electrodes was used with $1-\mathrm{cm}$ 
distance between their poles. They were placed following longitudinal direction of the fibers ${ }^{9}$ and were connected to the fist anterior region of the volunteers. Electric potentials of the anterior right and left a suprahyoid muscles were recorded during $2 \mathrm{~s}$ per procedure.

Recording started after stabilization of the electromyographic signal, according to the following protocol: a) continuous water suction using a straw during recording; b) two-min rest between records ${ }^{9}$; c) swallowing of $20 \mathrm{~mL}$ water after investigator's verbal command. These recordings were repeated 3 times. As reference muscle contraction, the electromyographic means obtained during dynamic activity were used. Data normalization was performed using the formula: rms values $(\mu \mathrm{V})$ during swallowing/ RMS values $(\mu \mathrm{V})$ during suction $\times 100^{20}$.

Data collected in the Stage 1 were analyzed statistically by Tukey-Kramer test (a evaluation), Kruskal-Wallis test (b evaluation) and Student's t-test (c evaluation); Data collected in the Stage 2 were analyzed statistically by ANOVA and Tukey-Kramer, except for the cases with nonnormal data distribution, which were analyzed by KruskalWallis non-parametric test. The tables elaboration have followed the same model as other works previously published by the same authors ${ }^{21}$.

\section{RESULTS}

Results from the oral myofunctional evaluation of group $\mathrm{C}$ will be reported comparatively to those of group $\mathrm{T}$, before and after first premolar extraction.

Oral myofunctional evaluation demonstrated, by visual inspection of lip posture at rest, that $76.4 \%$ of group $\mathrm{T} 1 \mathrm{had}$ the habitual open lip posture, while $23.5 \%$ had closed lips at habitual rest. After the orthodontic treatment, 52.9\% still had a habitual posture of open lips at rest, while $47.0 \%$ this group and $100 \%$ of group $\mathrm{C}$ had closed lip posture.

The interlabial relation of groups $\mathrm{T}$ and $\mathrm{C}$ at habitual lip rest posture showed that groups $\mathrm{T} 1$ and $\mathrm{T} 2$ had statistically similar habitual lip resting posture $(\mathrm{p}>0.05)$, differing significantly, however, from group $\mathrm{C}(\mathrm{p}<0.05)$ (Table 1$)$.

The evaluation of lip posture during swallowing showed that $58.8 \%$ of group T1 and a $100 \%$ group C presented lip seal, while $41.7 \%$ of group T1 presented interposition of the lower lip against the maxillary incisors. On the other hand, $100 \%$ of group T2 had lip seal during this function. Comparing group $\mathrm{C}$ to groups $\mathrm{T} 1$ and $\mathrm{T} 2$, data in Table 2 show that group $\mathrm{C}$ was statistically similar to group $\mathrm{T} 2$ $(\mathrm{p}>0.05)$, but differed significantly from $\mathrm{T} 1,(\mathrm{p}<0.05)$, since many of group $\mathrm{T}$ volunteers presented interposition of the lower lip against the maxillary incisors during swallowing. The values were statistically significant $(\mathrm{p}<0.05)$, when groups T1 and T2 were compared, as all volunteers presented lip seal during swallowing after the orthodontic treatment.

Mentalis muscle contraction was observed in $70.5 \%$ of group T1, while $64.7 \%$ of group T2 and $100 \%$ of group C did not show this contraction during swallowing (Table 3 ).
The maxillary and mandibular dental arch perimeters of groups $\mathrm{C}, \mathrm{T} 1$ and $\mathrm{T} 2$, were analyzed statistically by the Student's t-test (Table 4 and 5). It was observed that the maxillary dental arch perimeter of group $\mathrm{C}$ was similar to that of group T1, but differed to that of group T2, which was the smallest one. The mandibular dental arch perimeter of group $\mathrm{C}$ was significantly larger from those of groups T1 and T2,

TABLE 1- Means of habitual lip rest posture length (in $\mathrm{mm}$ ) of groups $\mathrm{C}, \mathrm{T} 1$ and $\mathrm{T} 2$

$\begin{array}{ll}\text { Group T1 } & 2.1402 \mathrm{~A} \\ \text { Group T2 } & 1.7695 \mathrm{~A} \\ \text { Group C } & 1.000 \mathrm{~B}\end{array}$

*Different letters indicate statistically significant difference at $5 \%$ (Tukey-Kramer test; D.M.S = 0.5730).

TABLE 2- Comparison among groups $\mathrm{C}, \mathrm{T} 1$ and $\mathrm{T} 2$ regarding lip posture during evaluation of clinical swallowing

\begin{tabular}{cl}
\hline \multicolumn{1}{c}{ Groups } & Value-p \\
\hline Group C X Group T1 & $\mathrm{p}<0.01^{*}$ \\
Group C X Group T2 & $\mathrm{NS}$ \\
Group T1 X Group T2 & $\mathrm{p}<0.01^{*}$ \\
\hline
\end{tabular}

NS = non-significant; ${ }^{*}$ Significant at $5 \%$ level (KruskalWallis test).

TABLE 3- Comparing among groups C, T1 and T2 regarding mentalis muscle contraction during swallowing

\begin{tabular}{cl}
\hline \multicolumn{1}{c}{ Groups } & Value $-\mathbf{p}$ \\
\hline Group C X Group T1 & $\mathrm{p}<0.001^{*}$ \\
Group C X Group T2 & $\mathrm{P}<0.001^{*}$ \\
Group T1 X Group T1 & $\mathrm{NS}$ \\
\hline
\end{tabular}

*NS = non-significant; * Significance at $5 \%$ level (KruskalWallis test).

TABLE 4- Maxillary and mandibular dental arch perimeters (means $\pm \mathrm{SD}$; in $\mathrm{mm}$ ) of groups $\mathrm{C}, \mathrm{T} 1$ and T2

\begin{tabular}{ll} 
Maxillary Arch Group C & $138.1176 \pm 8.46$ \\
Maxillary Arch Group T1 & $133.5882 \pm 9.47$ \\
Maxillary Arch T2 Group & $125.9412 \pm 7.23$ \\
Mandibular Arch Group C & $130.7647 \pm 8.15$ \\
Mandibular Arch Group T1 & $124.5882 \pm 8.89$ \\
Mandibular Arch T2 Group & $120.7059 \pm 6.61$ \\
\hline
\end{tabular}


which, in turn, did not differ significantly from each other (Tables 4 and 5).

Electromyographic data from the orbicularis oris muscle during the functions of group $\mathrm{C}$ were compared to those of group $\mathrm{T}^{23}$ (Table 6.), with no statistically significant differences $(\mathrm{p}>0.05)$, either in the upper or lower portions of the muscle. However, the upper portion differed significantly $(\mathrm{p}<0.05)$ from the lower portion, both in group $\mathrm{C}$ and in group T2.

TABLE 5- Comparison among groups C, T1 and T2 regarding maxillary and mandibular dental arch perimeters

\section{Treatment}

Significance*

MAC vs MAT1

MAC vs MAT2

MAT1 vs MAT2

MDAC vs MDAT1

MDAC vs MDAT2

MDAT1 vs MDAT2

MAC - maxillary arch group C; MDAC - mandibular arch group C; MAT1 - maxillary arch group T1; MDAT1 mandibular arch group T1; MAT2 - maxillary arch group T2; MDAT2 - mandibular arch group T2; NS: non-significant; ${ }^{*}$ Significant at level of $5 \%$ (Student's t-test).

TABLE 6- Comparison between group $\mathrm{C}$ and group T2 normalized data of orbicularis oris muscle swallowing by Kruskal-Wallis test.

$\begin{array}{ll}\text { UOM - Group C } & 110.32 \mathrm{~A} \\ \text { LOM - Group C } & 280.20 \text { B } \\ \text { UOM - Group T2 } & 92.38 \text { A } \\ \text { LOM - Group T2 } & 215.34 \text { B }\end{array}$

Different letters indicate statistically significant difference at $5 \%$. UOM: Orbicularis oris muscle upper portion; LOM: Orbicularis oris muscle lower portion.

\section{DISCUSSION}

First premolar extraction has been widely discussed since the 19 th century ${ }^{7}$. The pendulum first premolar "extraction or non-extraction" is one of the most discussed subjects in orthodontics as well as great matter of concern in dentistry due to the possibility of myofunctional alterations as a limiting or etiologic factor towards the orthodontic treatment, causing a lack of stability on this treatment.

The literature focuses on the intimate relationship existing between the shape and functions of the stomatognathic system, emphasizing the specific set of structural characteristics in each individual. This fact could be related to the infeasible lip seal due to the maxillomandibular unbalance and the maxillary incisor vestibule typical of Class II/1 patients ${ }^{5,8,17}$. In addition, because of its inclination, the upper lip lays on the palatal surface of the maxillary incisors at rest, adapting to their accentuated overjet, and providing the habitual open-lip posture while at rest. However, in this study, group C presented the habitual closed lip posture typical in Class I patients ${ }^{5}$ and group T2 showed a decrease on open lip posture at rest, revealing that premolar extraction can bring soft tissue improvement in some cases $^{3,6}$ as a consequence of the improvement on maxillary incisor position ${ }^{5}$.

Significant differences were observed between the habitual lip rest posture of groups T1 and T2 and that of group C, but the treatment groups had similar habitual lip rest posture to each other. It is due to the fact that the anterior tooth retraction value was not enough to cause lip seal in $52.9 \%$ of volunteers, modifying the soft tissue profile, while not promoting functional adequacy ${ }^{3,18,23}$. Other perpetuating factors of a poor lip posture, namely labial hypofunction $^{5,12,17,25}$, possible upper lip shortening and probable lengthening of the lower facial third ${ }^{25}$, are common characteristics of Class II/1 malocclusion.

Visual lip posture inspection during swallowing showed that $41.17 \%$ of group $\mathrm{T} 1$ volunteers presented lower lip interposition on maxillary incisors during this function, while all groups $\mathrm{C}$ and $\mathrm{T} 2$ volunteers had lip seal during this function. This fact demonstrates that lower lip interposition on maxillary incisors is probably a posture adaptation imposed by the poor interarch relationship observed in Class II/1 malocclusion before orthodontic treatment ${ }^{8,23}$.

The clinical analysis of the mentalis muscle showed statistically significant differences among groups C, T1 and $\mathrm{T} 2$, being contracted during swallowing in both treatment groups, and non-contracted in $100 \%$ of group $\mathrm{C}$ volunteers (Table 3). The fact that statistically significant differences were not observed between groups $\mathrm{T} 1$ and $\mathrm{T} 2$ seems to suggest that the greater activation of the lower portion of the orbicularis oris muscle related to its upper portion occurs due to mentalis muscle participation during activation of the lower portion of the orbicularis oris muscle. This difference indicates that the mentalis muscle had a great contribution during lip seal in group T. Even after orthodontic treatment and in cases of lip muscle hypofunction, there is major perioral muscle activation, since additional motion units are recruited to keep lip seal ${ }^{8}$.

First premolar extraction and anterior tooth retraction allowed an excessive overjet correction in group $\mathrm{T}$, making possible lip seal after treatment. After orthodontic treatment, there was a difference on dental arch perimeter (Tables 4 and 5). The maxillary dental arch perimeter of group $C$ was similar to that of group T1. This situation can be explained by the fact that Class II/1 malocclusion can present a trend to lower facial third increase and disharmony in the apical bone bases, in an anteroposterior direction ${ }^{5}$. This alters the maxillomandibular complex relation with the skull base ${ }^{16}$, differing from Class I. On the other hand, the maxillary arch 
in Class I subjects typically has a U-shaped geometry while in Class II/1 malocclusion this geometry is similar to a V letter, which suggests that the difference of arch shapes does not modify their measures because there is a similar number of teeth in both arches and normally there is no crowding in the maxillary arch. This hypothesis seems to support the statistical similarity observed between the groups with respect to their maxillary dental arch perimeters.

On the other hand, group T1 presented significantly smaller mandibular dental arch perimeter than group C (Table 4 and 5). This result can be attributed to the fact that crowding was frequently observed in group $\mathrm{T} 1$ subjects and, in some cases, the lower lip caused lingual tipping of the mandibular anterior teeth, which could have decreased the arch perimeter. This condition was not observed in Class I subjects (group C).

Comparing the maxillary and mandibular dental arch perimeters of group $\mathrm{C}$ to $\mathrm{T} 2$, and maxillary dental arch perimeters of groups $\mathrm{T} 1$ and $\mathrm{T} 2$, statistically significant differences were observed, as shown on Table 4 . The treated groups showed a decrease in arch measurements, occurred due to tooth retraction for correction of Class II/1 $1^{3,6}$. The mandibular dental arches of groups $\mathrm{T} 1$ and $\mathrm{T} 2$ did not differ significantly (Table 4) because some volunteers did not have their mandibular premolars extracted during orthodontics, according to the treatment plan.

On the other hand, electromyographic studies have demonstrated that the orbicularis oris muscle has a greater activity in Class II/1 subjects with incompetent lips, during suctioning and swallowing, compared to subjects who had competent lips ${ }^{2,11,14,24}$. The findings of these studies show that subjects with longer lower facial third and/or anteroposterior maxillomandibular discrepancy are more prone to have increased perioral muscle activity and labial incompetence.

Subjects with atypical swallowing, during deglutition of saliva and water, have greater electromyographic activity of perioral muscles and smaller activity of masticatory muscles when compared to clinically normal volunteers. None of these pattern alterations is observed after the orthodontic treatment ${ }^{22}$.

Class II/1 malocclusion subjects have been shown to present smaller activity of the upper portion of the orbicularis oris muscle during mastication and swallowing and hyperactivity of the lower portion of the orbicularis oris muscle even at rest ${ }^{15}$. In the present study, there were no statistically significant differences between groups $\mathrm{C}$ and T2 regarding the electromyographic value of the lower and upper portions of the orbicularis oris muscle. The decrease of the dental arch perimeter produced by first premolar extraction seemed to benefit lip seal, unlike the findings of Stormen \& Panchez (1999).

Further research on patients with normal occlusion and malocclusion is needed to confirm these results because the mentalis muscle was not evaluated electromyographically in this study due to methodological difficulties. In view of the results hereby discussed, it seems that the perioral musculature was benefited by the orthodontic treatment, as lip seal occurred during the evaluated functions; during habitual rest, however, these muscles remained hypoactive. These results suggest that the desired long-term orthodontic stability ${ }^{2,7,18,19}$ can be threatened by non-treated myofunctional alterations after removal of orthodontic appliances, making recurrence possible ${ }^{2,18}$, mainly if related to the modiolus region, which is located above the premolars and is considered as force redistributing region ${ }^{13}$.

\section{CONCLUSIONS}

First premolar extraction in Class II/1 malocclusion treatment caused a decrease of dental arch perimeter. Class II/1 correction by first premolar extraction seemed to motivate lip seal only during swallowing. The oral myofunctional alterations found after orthodontic treatment in Class II/1 cases seemed to jeopardize the long-term orthodontic stability, making recurrence possible. Oral myofunctional evaluation and treatment supportive to orthodontics are of paramount importance to maintain or restore the myofunctional balance of the stomatognathic system.

\section{REFERENCES}

1- Acierno SP, Baratta RV, Solomonow M. A practical guide to electromyography for biomechanics. New Orleans: Louisiana State University; 1995.

2- Ahlgren J. EMG studies of lip and cheek activity in sucking habits. Swed Dent J. 1995;19(3):95-101.

3- Anderson BD. Multiple extraction patterns in severe discrepancy cases. Angle Orthod. 1975;45(4):291-303.

4- Basmajian JV, De Luca CJ. Muscle alive: their function revealed by electromyography. 5th ed. Baltimore: Williams and Wilkins; 1985.

5- Bianchini EMG. A cefalometria nas alterações miofuncionais orais: diagnóstico e tratamento fonoaudiológico. São Paulo: Prófono; 1995.

6- Bishara SE, Cummins DM, Jakobuen JR. Treatment and posttreatment changes in patients with Class II/1, malocclusion after extraction and nonextraction treatment. Am J Orthod Orthop. 1997;111(1):18-27.

7- Case CS. The question of extraction in orthodontia Trans NDA 1911.(reprinted) Am J Orthod. 1964;50(1):658-691.

8- Cleal JF. Deglutition a study of form and function. Am J Orthod. 1965;51(1):566-94.

9- De Luca CJ. The use of surface electromyography in biomechanics. J Appl Biomech. 1997;13(1):135-63.

10-Drobocky OB, Smith RJ. Changes in facial profile during orthodontic treatment with extraction of four first premolars. Am J Orthod. 1989;95(3):220-30.

11 - Gustaffson M, Ahlgren J. Mentalis and orbicularis oris activity in children with incompetent lips on electromyographic and cephalometric study. Acta Odont. Scand. 1975;33(1):355-63. 
12 - Hanson ML, Barret RH. Fundamentals of orofacial mycology. Illinois: Charles Thomas; 1988.

13- Lightoller GHS. Facial muscles. The modiolus and muscles surrounding the rima oris with some remarks about panniculus adiposus. J Anat. 1925;60(1):1-85

14- Lowe AA, Takada K. Associations between anterior temporal, masseter and orbiculares oris muscle activity and craniofacial morphology in children. Am J Orthod. 1984;86(4):319-30.

15-Marchiori SC, Vitti M. Estudo eletromiográfico do músculo orbicular da boca em indivíduos com oclusão normal e maloclusões. RGO. 1996;44(6):331-334.

16- Pfeiffer JP, Grobét D. A philosophy of combined orthopedicorthodontic. Am J Orthod. 1982;81(3):185-201.

17- Prabhu NT, Munshi AK. Ultrasonographic observation of the circumoral musculature: an in-vivo study. J Clin Pediatr Dent. 1995;19(3):195-203.

18- Proffit W. Forty-year review of extraction frequencies at a university orthodontic clinic. Angle Orthod. 1994;64(6):407-14.

19- Salzmann JA. An evaluation of retention and relapse following orthodontic therapy. Am J Orthod. 1965;51(10):779-81.

20 - Soderberg GL, Knutson LM. A guide of use and interpretation of knesiologic electromyographic date. Phys Ther. 2000;80(5):485-

21 - Souza DR, Semeghini TA, Kroll LB, Berzin F. Oral myofunctional and electromyografic evaluation of the anterior suprahyoid muscles and tongue thrust in patients with class II/1 maloclusion submitted to first premolar extraction. J Appl Oral Sci. 2007;15(1):24-8.

22- Stormer K, Pancherz H. Electromyography of the perioral and masticatory muscles in orthodontic patients with atypical swallowing. J Orofac Orthop. 1999;60(1):13-23.

23 - Subtelny JD. Malocclusions, orthodontic corrections and orofacial muscle adaptation. Angle Orthod. 1970;3(1):170-201.

24- Tosello DO, Vitti M, Bérzin F. EMG activity of the orbicularis oris and MENTALIS muscles in children with malocclusion, incompetent lips and atypical swallowing. Part I. J Oral Rehabil 1998;25(1):838-46.

25- Yogosawa F. Predicting soft tissue profile changes concurrent with orthodontic treatment. Angle Orthod. 1990;60(3):199-206.

26- Zemlin WR. Speech and hearing science: anatomy and physiology. Allyn \& Bacon; 1998. 